\title{
Asxl1 exerts an antiproliferative effect on mouse lung maturation via epigenetic repression of the E2f1-Nmyc axis
}

Seungtae Moon', Sun-Kyoung Im², Nackhyoung Kim', Hyesook Youn ${ }^{1}$, Ui-Hyun Park', Joo-Yeon Kim³, A.-Reum Kim', So-Jung $\mathrm{An}^{1}$, Ji-Hoon Kim", Woong Sun $\mathbb{B}^{3}$, Jin-Taek Hwang ${ }^{5}$, Eun-Joo Kim and Soo-Jong Um

\begin{abstract}
Although additional sex combs-like 1 (ASXL1) has been extensively described in hematologic malignancies, little is known about the molecular role of ASXL1 in organ development. Here, we show that Asx/1 ablation in mice results in postnatal lethality due to cyanosis, a respiratory failure. This lung defect is likely caused by higher proliferative potential and reduced expression of surfactant proteins, leading to reduced air space and defective lung maturation. By microarray analysis, we identified E2F1-responsive genes, including Nmyc, as targets repressed by Asxl1. Nmyc and Asx/1 are reciprocally expressed during the fetal development of normal mouse lungs, whereas Nmyc downregulation is impaired in Asx/1-deficient lungs. Together with E2F1 and ASXL1, host cell factor 1 (HCF-1), purified as an Asxl1bound protein, is recruited to the E2F1-binding site of the Nmyc promoter. The interaction occurs between the Cterminal region of Asxl1 and the N-terminal Kelch domain of HCF-1. Trimethylation (me3) of histone H3 lysine 27 (H3K27) is enriched in the Nmyc promoter upon Asx/1 overexpression, whereas it is downregulated in Asx/1-deleted lung and -depleted A549 cells, similar to H3K9me3, another repressive histone marker. Overall, these findings suggest that Asxl1 modulates proliferation of lung epithelial cells via the epigenetic repression of Nmyc expression, deficiency of which may cause hyperplasia, leading to dyspnea.
\end{abstract}

\section{Introduction}

The additional sex combs-like (ASXL) family is the mammalian homolog of the additional sex combs $(A s x)$ gene, which is an enhancer of trithorax group (TrxG) and polycomb group (PcG) proteins in Drosophila. There are three members of the $A S X L$ family-ASXL1, ASXL2, and $A S X L 3^{2}$. ASXL family proteins are chromatin factors that exert diverse effects, including on tumor suppression and development, by modulating gene expression. We previously demonstrated that ASXL1 is primarily located in the nucleus and regulates nuclear hormone receptors

\footnotetext{
Correspondence: S.-J. Um (umsj@sejong.ac.kr)

${ }^{1}$ Department of Integrative Bioscience and Biotechnology, Sejong University, Seoul 05006, Korea

${ }^{2}$ Severance Biomedical Science Institute, Yonsei University College of Medicine, Seoul 06273, Korea

Full list of author information is available at the end of the article

Edited by M. Herold
}

in cooperation with either SRC1 as a coactivator or HP1 $\alpha$ as a corepressor ${ }^{3,4}$. Drosophila Asx complexes with the histone deubiquitinase Calypso (mammalian BAP1) forms a polycomb repressive deubiquitinase (PR-DUB) complex and stimulates removal by Calypso of monoubiquitin from H2A lysine 119 for transcriptional repression ${ }^{5}$. The enzymatic activity of the PR-DUB complex was also examined in mammals using the ASXL family ${ }^{6}$. Additionally, ASXL1 interacts with EZH2, a member of polycomb repressive complex 2 (PRC2) and responds to PRC2-mediated gene silencing ${ }^{7,8}$.

ASXL1 is frequently mutated in hematological or myeloid malignancies; such mutations are associated with adverse outcomes $^{9-11}$. In contrast, ASXL1 mutations in solid tumors are rarely reported, and their effects are unclear $^{2}$. Hematopoietic-specific deletion of Asxl1 or overexpression of an ASXL1 mutant resulted in

\section{(c) The Author(s) 2018}

(c) (i) Open Access This article is licensed under a Creative Commons Attribution 4.0 International License, which permits use, sharing, adaptation, distribution and reproduction cc) in any medium or format, as long as you give appropriate credit to the original author(s) and the source, provide a link to the Creative Commons license, and indicate if changes were made. The images or other third party material in this article are included in the article's Creative Commons license, unless indicated otherwise in a credit line to the material. If material is not included in the article's Creative Commons license and your intended use is not permitted by statutory regulation or exceeds the permitted use, you will need to obtain permission directly from the copyright holder. To view a copy of this license, visit http://creativecommons.org/licenses/by/4.0/. 
myelodysplasia-like syndromes in mice ${ }^{12-15}$. Despite reports of somatic ASXL1 mutations in leukemia, the mechanism by which ASXL1 exerts effects against cancer is unknown. Asxl1 ablation leads to significant reduction of H3K27 trimethylation, likely due to impaired interaction with Ezh2, a histone methyltransferase ${ }^{12}$. Although Bap1 loss also promotes myeloid transformation in mice $^{16}$, the underlying mechanism appears to be different from that of Asxl1 loss in terms of PRC2 regulation ${ }^{17}$. Germline ASXL1 mutations are reported in around 50\% of patients with Bohring-Opitz syndrome ${ }^{18,19}$. Recent studies using Asxl1-null mouse models indicated a critical role for Asxl1 in development. Depending on the model, Asxl1 loss causes embryonic lethality and developmental abnormalities, including dwarfism, anophthalmia, microcephaly, kidney podocyte defects, and craniofacial defects $^{12,14,20,21}$. Other investigation using Asxl1-null mice reported the potential role of Asxl1 in lung maturation $^{22}$. However, the molecular mechanism by which Asxl1 loss causes these defects remains unexplored. Recently, we showed that Asxl1 interacts with Akt in mouse embryonic fibroblasts (MEFs) to promote cell proliferation, and Asxl1 disruption results in cellular senescence by increasing p16Ink4a expression via Ezh2 inactivation $^{8}$. Intriguingly, this role of Asxl1 in MEF proliferation is the opposite of its function as a tumor suppressor ${ }^{23}$. Therefore, the mechanism underlying this difference in various tissues and at different developmental stages needs to be investigated.

Lung development in mice is divided into five distinct stages according to gestational age: embryonic (E9-11.5), pseudoglandular (E11.5-16.5), canalicular (E16.5-17.5), saccular (E17.5-PN5), and alveolar (PN5-28) stages $^{24}$. During lung maturation, the respiratory air spaces are formed by progressive branching of epithelial-lined airways into the lung mesenchyme ${ }^{25}$. Epithelial cells lining the pulmonary air spaces begin to differentiate into functional Type I and Type II pneumocytes ${ }^{26}$. Type I pneumocytes are squamous and thin, cover about $95 \%$ of epithelial air surface, and participate in gas exchange, whereas Type II pneumocytes are granular and cuboidal, and secrete surfactants accompanying with depletion of glycogen content. Genetic and epigenetic defects in these pneumocytes may cause lung diseases including respiratory distress syndrome (RDS) and cancer. Our previous observations that most newborn Asxl1-null mice died just after birth prompted us to investigate the underlying mechanism, focusing on the role of Asxl1 in lung development associated with epithelial cell proliferation and differentiation.

Our studies indicated that the dyspnea in $A s x l 1^{-1-}$ mice is caused by abnormally increased proliferation and reduced maturation of Type 11 pneumocytes. The underlying mechanism was identified by microarray analysis,
ASXL1-bound complex purification, transcription assay, and chromatin immunoprecipitation. Overall, our data suggest that Asxl1 forms a complex with HCF-1 to repress E2F1-driven Nmyc transcription by regulating trimethylation of histone $\mathrm{H} 3$ lysine 27 and thus controls the Nmyc-associated proliferation of lung epithelial cells.

\section{Results \\ Asxl1-knockout mice show perinatal lethality due to respiratory failure}

To determine the physiological function of Asxl1, we generated Asxl1 homozygous mice $\left(A s x l 1^{-1-}\right)$ (Supplementary Figure S1a $)^{21}$. Inactivation of the $A s x l 1$ gene was confirmed by Western blotting (WB) (Supplementary Figure S1b) and reverse transcription-quantitative polymerase chain reaction (RT-qPCR) (Supplementary Figure S1c). Before birth, embryos from Asxl1 ${ }^{+l-}$ intercrosses showed the expected Mendelian ratio (Supplementary Table S1). However, Asxl1 ${ }^{-1-}$ mice died within $1.5 \mathrm{~h}$ of birth due to respiratory failure (Supplementary Figure S1d). In addition to cyanosis, $A s x l 1^{-1-}$ neonates showed a small body and lung size, anophthalmia, microcephaly, and cleft palates compared to wild-type (WT) neonates after birth (Supplementary Figure S1a, e, and f). Considering the intact structure of the trachea and heart in mutant mice (Supplementary Figure $\mathrm{S} 1 \mathrm{~g}-\mathrm{i}$ ), we hypothesized that the respiratory failure arose from defects in lung development. The lung of a WT neonate was well-inflated and floated in phosphatebuffered saline (PBS), whereas the Asxl1 $1^{-/-}$lung sank (Fig. 1a). As shown by hematoxylin and eosin (H\&E) staining, the homozygous $A s x l 1^{-1-}$ lung failed to inflate with air because of its thicker alveolar wall (Fig. 1b), smaller air space, and more numerous small alveoli (Fig. 1c) compared to the WT or the heterozygote. Anatomical changes in alveoli were analyzed from E14.5 to P0 during embryonic lung development. Until E16.5, no significant difference between the WT and $A s x l 1^{-1-}$ mice was observed. However, alveoli of the $A s x l 1^{-1-}$ lung at E18.5 and P0 showed a remarkably reduced aerated space, which was compacted with immature-looking cuboidal cells (Fig. 1d). In situ hybridization (ISH) using an E18.5 embryo indicated that Asxl1 was expressed throughout the lung epithelium and mesenchyme region (Fig. 1e and Supplementary Figure S1j). Insertion of the $L a c Z$ cassette in the Asxl1 allele allows to monitor the expression of Asxl1 gene by X-gal staining ${ }^{21}$. Mutant lungs at various embryonic stages were stained at both epithelium and mesenchyme regions, suggesting ubiquitous expression of Asxl1 during lung development (Supplementary Figure S1k). This Asxl1 expression pattern was further confirmed using isolated epithelial and mesenchymal cells at E18.5 (Supplementary Figure S1l), and their markers Ccsp and Vimentin (Supplementary Figure S1m). Overall, these 


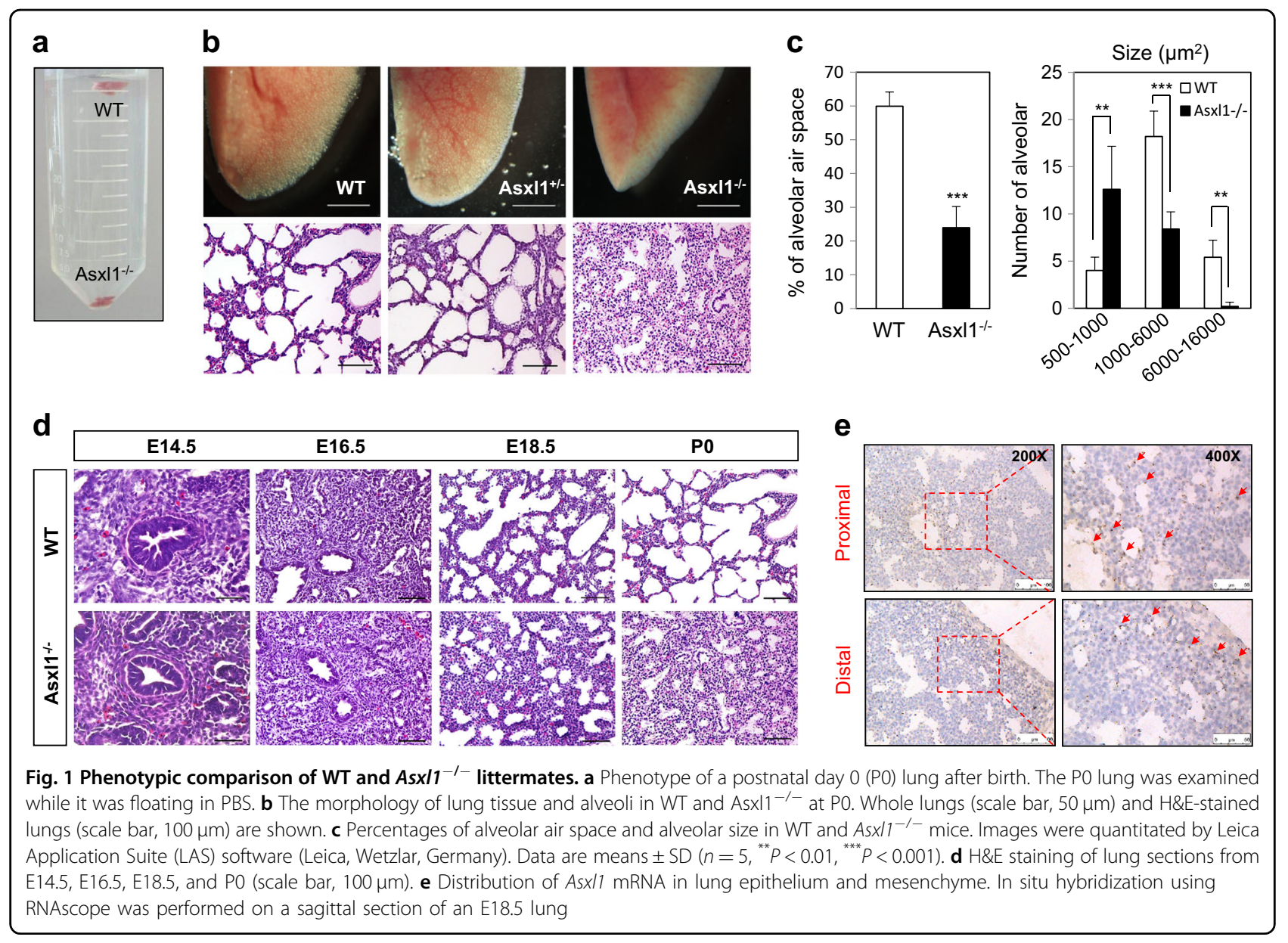

data suggest that Asxl1, expressed in lung, is critical for proper alveolar formation in the saccular phase of lung development.

Asxl1 loss increases the proliferation of lung epithelial cells

Abnormality of lung alveolar structure might be related to altered cell proliferation, apoptosis, or differentiation. Cell proliferation was measured by staining for the two proliferation markers Ki-67 and proliferating cell nuclear antigen (PCNA). The protein and mRNA levels of Ki-67 and PCNA in Asxl1-deleted lungs were significantly higher than those in WT lungs (Fig. 2a, b), but there was no difference between the distal and proximal regions (Supplementary Figure S2a, b). No significant change in apoptosis was detected by terminal deoxynucleotidyl transferase dUTP Nick-End Labeling (TUNEL) assay (Supplementary Figure S2c, d), suggesting that the thickened alveolar wall results from the increased proliferation. Further co-immunostaining with antibodies against PCNA and a lung epithelial marker Nkx2.1 indicated that higher proliferation rate in Asxl1-null lung is likely due to the increased proliferation of epithelial cells (Supplementary Figure S2e). To examine the role of Asxl1 ablation in the differentiation of airway cells, the expression of Club cell secretory protein (Ccsp, also known as Cc10, an airway epithelial marker) and alpha smooth muscle actin ( $\alpha \mathrm{Sma})$ was monitored. As shown in Fig. $2 \mathrm{c}$, the numbers of Ccsp-positive airway epithelial cells were dramatically decreased in E18.5 Asxl1 ${ }^{-1-}$ lung, whereas no significant change in those positive for the muscle marker $\alpha$ Sma was observed. The mRNA level of Ccsp was also downregulated at E18.5 (Fig. 2d) and P0 (Supplementary Figure S2f). In addition, we observed the upregulation of Sox9, a marker of distal epithelial progenitor cells, in Asxl1-null E18.5 lung by immunostaining (Supplementary Figure S2g) and RT-qPCR (Supplementary Figure S2h). It has been reported that Sox9 downregulation starting at E16.5 is concurrent with terminal differentiation of Type I and II alveolar cells ${ }^{27}$. Further ex vivo organ culture using E10.5 and E12.5 lungs indicated no effect of Asxl1 ablation on branching morphogenesis (Supplementary Figure S2i, j). Overall, these data suggest that the defective phenotype of Asxl1-null lungs is due to the abnormally increased proliferation of epithelial cells, associating with dysregulated expression of Ccsp and Sox9. 
a

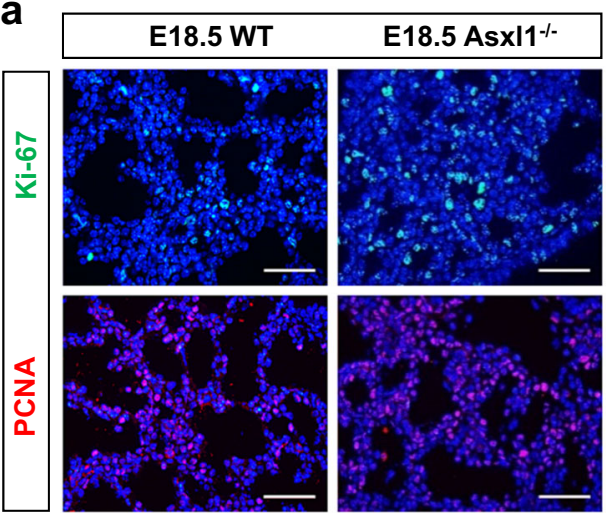

C

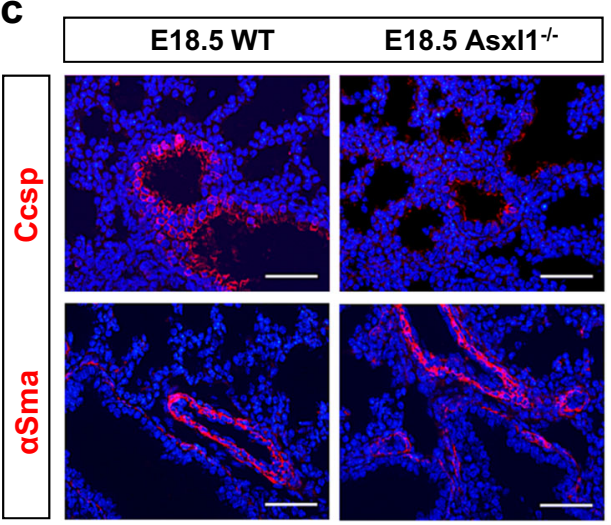

b

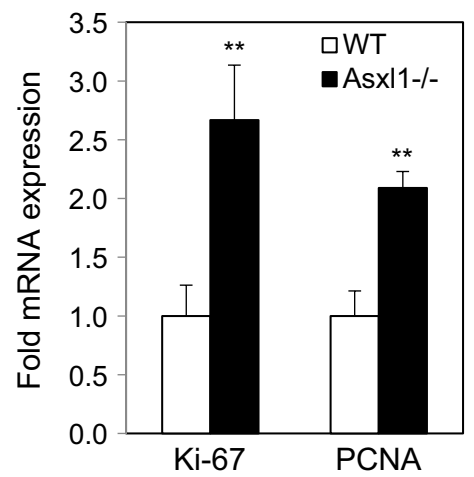

d

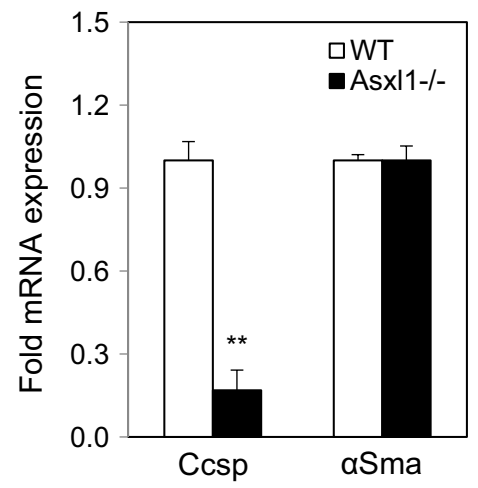

Fig. 2 Effect of Asxl1 on the proliferation of lung epithelial cells. a Cell proliferation was determined by immunostaining with Ki-67 and PCNA at E18.5. Scale bar, $100 \mu \mathrm{m}$. b mRNA expression levels were measured by RT-qPCR. c Differentiation was assayed using antibodies against the lung epithelial marker Ccsp and the muscle marker aSma at E18.5. $\mathbf{d}$ mRNA expression levels are shown. Data are means \pm SD $\left(n=6,{ }^{* * *} P<0.01\right)$

\section{Asxl1 is critical for lung alveolar maturation}

The dysregulated expression of Ccsp and Sox9 in Asxl1null lungs prompted us to investigate the role of Asxl1 in lung maturation using other epithelial differentiation markers. The WT lung at E18.5 showed welldifferentiated Type I and II pneumocytes expressing $\mathrm{T} 1 \alpha(P d p n)$ and surfactant protein-B (SP-B, coded from $S f t p b$ gene), whereas the $A s x l 1^{-1-}$ lung was compacted with precursor cells lacking Pdpn and SP-B expression, but heavily expressing proSP-C (Fig. 3a). Subsequent RTqPCR analysis revealed a significant reduction in the levels of Pdpn, Aqp5, Sftpb, and Sftpd (Fig. 3b). During the morphological and functional maturation of Type II cells, the reserved glycogen is converted into phospholipids to produce lamellar bodies, which are composed of surfactant lipids and proteins ${ }^{28}$. The increased proSP-C staining in $A s x l 1^{-1-}$ lungs indicated immature Type II pneumocytes (Fig. 3a). To confirm this, we performed periodic acid-Schiff (PAS) staining, which enables visualization of glycogen-rich cells. Indeed, the number of PAS-positive cells in the alveolar epithelium of the $A s x l 1^{-1-}$ lung was greater than that in the WT lung (Fig. 3c). Additionally, the percentage of glycogen-rich cells in the proximal and distal regions of the $A s x l 1^{-1-}$ lung was twofold higher than that in the WT control lung (Supplementary Figure S3a, b). Further, phospholipid deposits, which are indicative of Type II cell maturation, were assayed by Sudan Black B (SBB) staining (Fig. 3c). Consistent with the higher glycogen staining in $A s x l 1^{-1-}$ lung, less phospholipid staining was observed, suggesting that Type II pneumocytes in $A s x l 1^{-1-}$ alveoli are defective in maturation. Finally, this abnormal maturation was confirmed by measuring the expression of genes involved in lung lipid homeostasis. These genes were significantly downregulated in $A s x l 1^{-1-}$ lungs (Fig. 3d). Overall, we conclude that Asxl1 plays an essential role in alveolar maturation during fetal lung development.

\section{E2F1-responsive genes, including Nmyc, are targets of Asxl1}

To determine whether Asxl1 loss alters lung gene expression profiles, we performed a microarray analysis of WT and $A s x l 1^{-1-}$ E18.5 lungs. Subsequent analysis of the expression profiles identified 1307 genes, comprising 

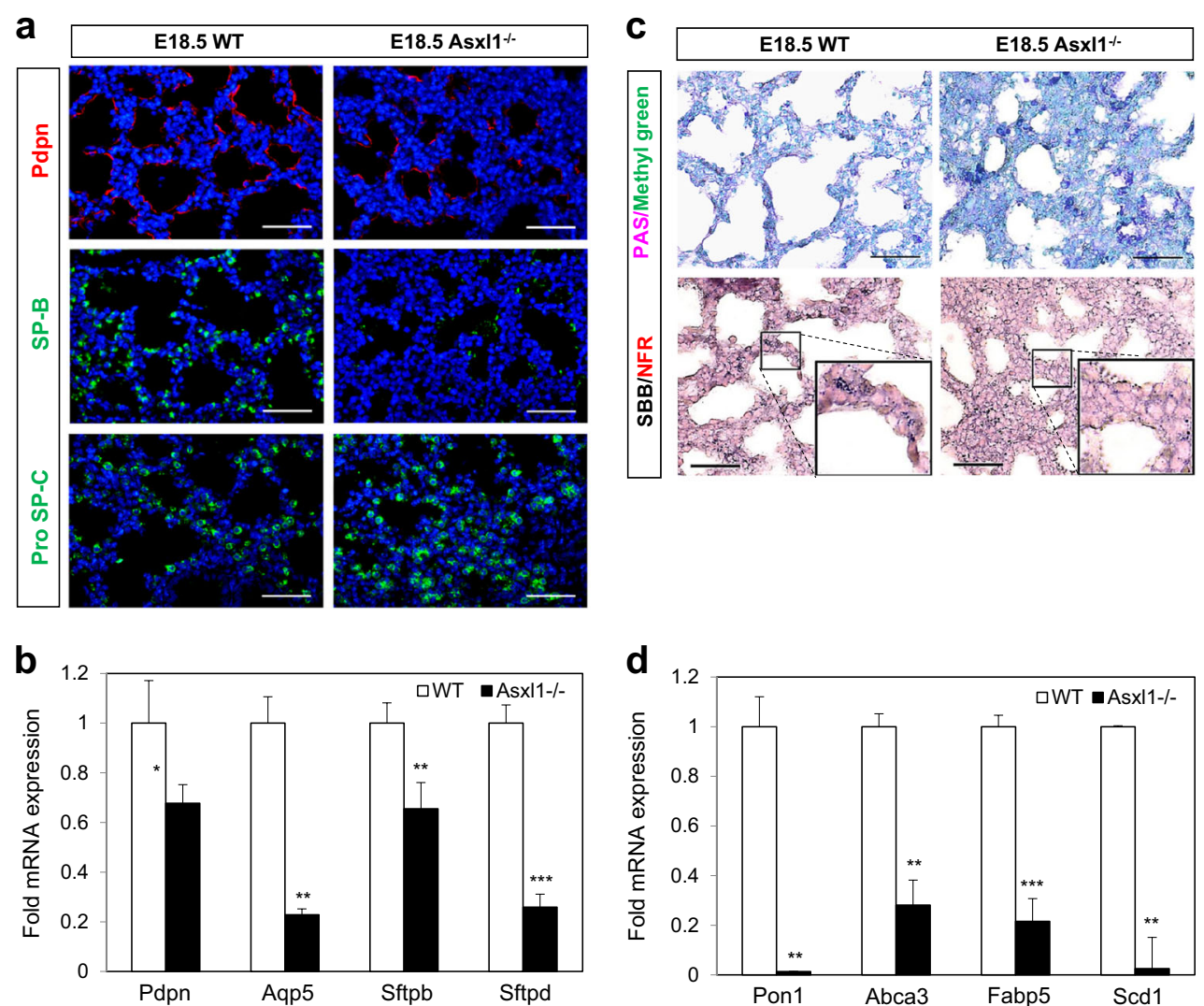

Fig. 3 Effects of Asxl1 on the maturation of alveolar Type I and Type II epithelial cells. a Immunostaining for mature Type I (Pdpn), Type II (SP-B), and immature Type II (proSP-C) markers at E18.5. Scale bar, $100 \mu \mathrm{m}$. b mRNA levels of Type I and Type II cell markers at E18.5 determined by RT-qPCR. Data are means \pm SD $\left(n=3,{ }^{*} P<0.05,{ }^{* * *} P<0.01,{ }^{* * *} P<0.001\right)$. c Periodic acid-Schiff (PAS) and Sudan Black B (SBB) staining. Scale bar, $100 \mu \mathrm{m}$. d Quantification of phospholipid homeostasis markers by RT-qPCR. Data are means \pm SD $\left(n=3,{ }^{* *} P<0.01,{ }^{* * *} P<0.01\right)$

1289 upregulated and 18 downregulated genes, with a greater than twofold change in expression in Asxl1-null lungs, strongly suggesting a role for Asxl1 in gene repression. Among these genes, the expression levels of cell cycle-, cell proliferation-, and apoptosis-associated genes were significantly altered (Supplementary Table S2 and Supplementary Figure S4a). Clustering analysis of cell proliferation-related genes showed that E2f1 target genes are highly upregulated in $A s x l 1^{-/-}$lungs (2.0 extension Plu0.05) (Fig. 4a). Further gene set enrichment analysis (GSEA) supported a repressive role for Asxl1 in the expression of genes associated with cell proliferation and E2f1 target genes (Supplementary Figure S4b, c). Upregulation of the mRNA levels of various E2f1 target genes, including Nmyc, in E18.5 Asxl1 $1^{-1-}$ lungs, was confirmed by RT-qPCR (Fig. 4b and Supplementary Figure S4d). Additionally, upregulation of the Nmyc mRNA level in Asxl1-deleted lungs was demonstrated by ISH (Fig. 4c). These observations were reproduced in ASXL1-depleted A549, adenocarcinomic human alveolar basal epithelial cells, using adenoviral expression of shASXL1 (Supplementary Figure S4e). However, Asxl1 overexpression by adenoviral infection of Flag-Asxl1 in A549 cells resulted in downregulation of some E2F1 target genes, including $N m y c$, but it did not affect the expression of $C D C 25 A$ and CDC6 (Supplementary Figure S4f). Microarray analysis identified Nmyc as one of the upregulated genes associated with cell cycle progression upon Asxl1 disruption (Fig. 4d). Nmyc is also a target of E2F1 in neuroblastomas $^{29}$. Nmyc overexpression or conditional deletion is driven by the surfactant $S P-C$ promoter/enhancer in mouse lung epithelium, and subsequent genome-wide approaches have provided information on the role of Nmyc in lung development at the transcriptional level ${ }^{30}$. A tight correlation between Nmyc and Asxl1 is suggested by the upregulation of $N m y c$ in $A s x l 1^{-1-}$ lungs, the similarities in the phenotypes of Nmyc-overexpressing and Asxl1-deleted lungs, and the GSEA of Nmyc-target genes (Supplementary Figure S4g). To support this observation, we compared our microarray data with those of previous studies using Nmyc transgenic (TG) and knockout (KO) mice $^{30,31}$. The expression pattern of genes upregulated in $A s x l 1^{-/-}$lungs was opposite to that of genes regulated by Nmyc KO but similar to genes regulated by 


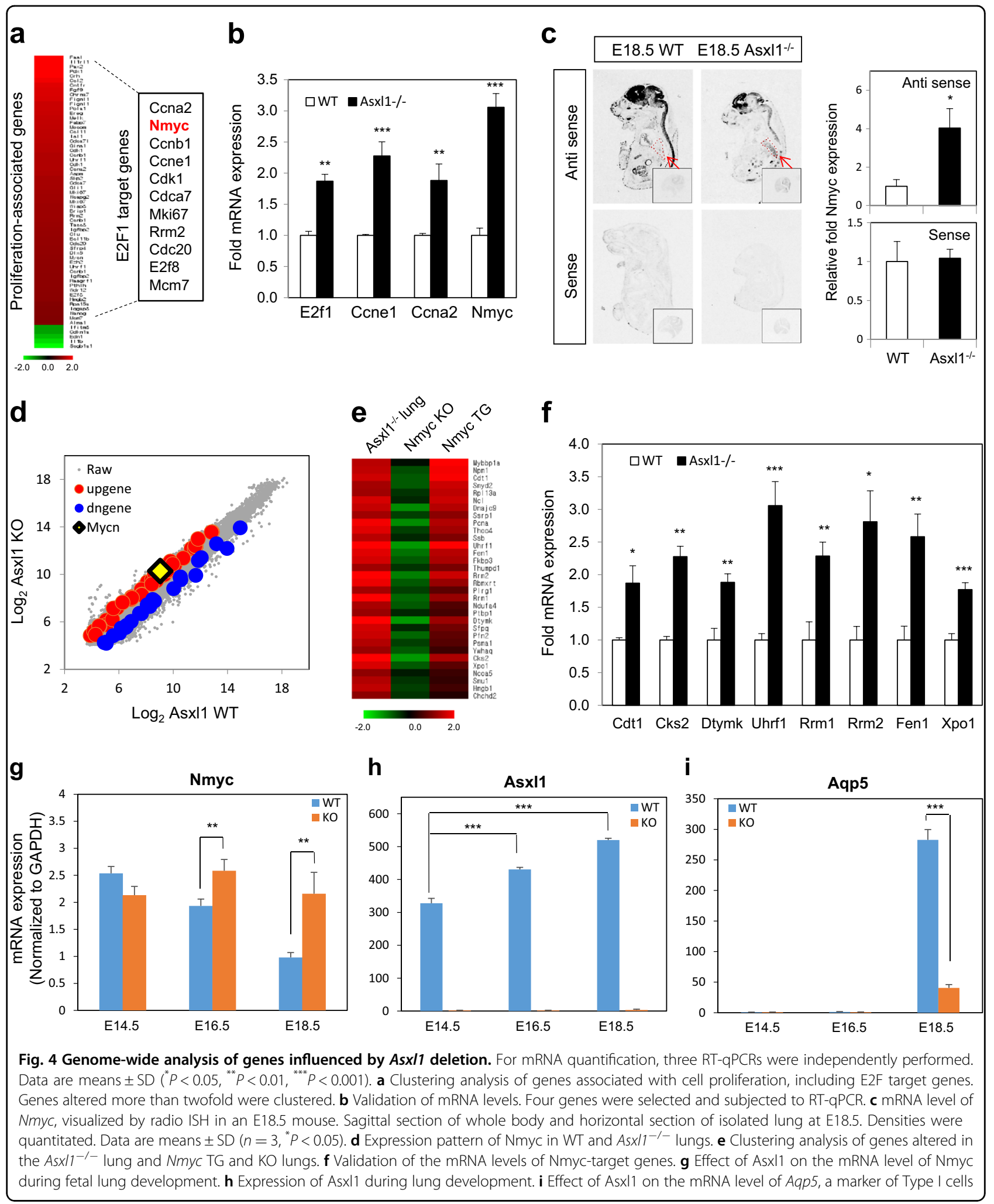

Nmyc overexpression (Fig. 4e and Supplementary Table S3). Significant upregulation of Nmyc-target genes in $A s x l 1^{-1-}$ lungs was determined by RT-qPCR (Fig. 4f).
As reported ${ }^{30}$, Nmyc expression declines from E14.5 to E18.5 during normal lung development, whereas Nmyc expression was elevated in Asxl1-deleted lungs (Fig. 4g). 
In contrast to Nmyc, Asxl1 expression increases from E14.5 to E18.5 (Fig. 4h). Downregulation of Nmyc is accompanied by upregulation of markers of differentiated epithelial cells (Aqp5, Sftpa, Sftpb, and Sftpc), which were downregulated in Asxl1-deleted lungs compared to WT lungs (Fig. 4i and Supplementary Figure S4h). Taken together, these data suggest that the expression of Nmyc is repressed by Asxl1 in the fetal lung at late stages of development.

\section{ASXL1 represses Nmyc expression via the E2F1-binding site}

Given that E2f1 target genes are upregulated by $A s x l 1$ disruption (Fig. 4a) and Nmyc is a target of E2F1, we determined whether ASXL1 inhibits Nmyc expression via the E2F1-binding site in the Nmyc promoter/enhancer. To answer this question, we generated luciferase reporter plasmids containing the WT and a mutant E2F1-binding site (Supplementary Figure S5a). As shown by luciferase assay using HEK293T cells transfected with Flag-tagged E2F1, the mutant with a defective E2F1-binding site failed to mediate an E2F1 response (Fig. 5a). To confirm whether E2F1 binds to putative binding site located in the region between -233 and -149 , chromatin immunoprecipitation (ChIP) assays were performed using primers specific for E2F1-binding site (site \#1) and covering negative control region (site \#2). We observed that the site \#1 is occupied by Flag-tagged E2F1 in two human non-small-cell lung cancer cell lines, H460 and A549 (Fig. 5b). As expected, Asxl1 overexpression reduced the luciferase activity in a dose-dependent manner (Supplementary Figure S5b). Further, we demonstrated that the intact E2F1-binding site is required for ASXL1-mediated repression (Fig. 5c), likely through ASXL1 binding to the site (Fig. 5d). ASXL1 occupancy of the E2F1-responsive sites was further confirmed using three other E2F1 target genes (CDC6, CCNA2, and CDC25A) in two lung cell lines (Supplementary Figure S5c, d).

\section{ASXL1 forms a complex with host cell factor (HCF)-1, a known binding partner of E2F1}

To assess how ASXL1 mediates transcriptional repression of E2F1 target genes, including $N m y c$, we identified ASXL1-interacting proteins. For this purpose, human HEK293T cells were engineered to overexpress Flag-tagged Asxl1. Affinity-purified Asxl1 complex a
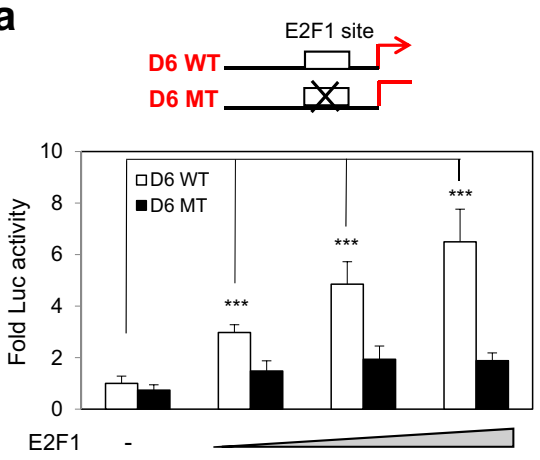

C

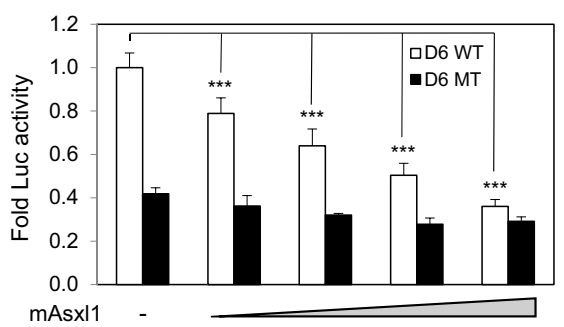

b
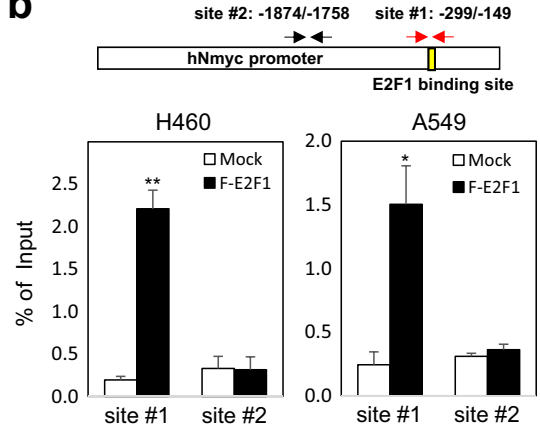

d
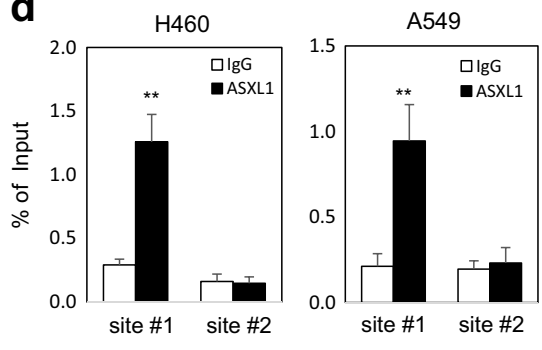

Fig. 5 Asxl1 represses Nmyc expression. For quantification, three independent experiments were performed. Data are means \pm SD $\left(^{* *} P<0.01,{ }^{* * *} P\right.$ $<0.001$ ). a Effect of E2F1 on Nmyc promoter activation. The WT (D6 WT) and mutant (D6 MT) E2F1-responsive sites in the Nmyc promoter are indicated. HEK293T cells were transfected with an Nmyc promoter-driven luciferase reporter gene and E2F1 expression vector (0, 0.01, 0.05, or 0.1 $\mu$ g). b E2F1 binding to the E2F1-responsive site in the Nmyc promoter. Schematic depiction of primer pairs to map the E2F1-binding site (shown by yellow). ChIP assay using an anti-Flag antibody and primer set for the human Nmyc promoter in H460 and A549 cells transfected with Flag-E2F1. Chromatin binding is shown as a percentage of the input. c Effect of Asxl1 on Nmyc promoter repression. HEK293T cells were transfected with the WT or mutant Nmyc luciferase reporter gene and ASXL1 (0.2, 0.4, 0.6, and 0.8 $\mu \mathrm{g})$. d ASXL1 binding to the Nmyc promoter. ChIP-qPCR was performed using an anti-ASXL1 antibody and primer set for Nmyc promoter in A549 and H460 cells 


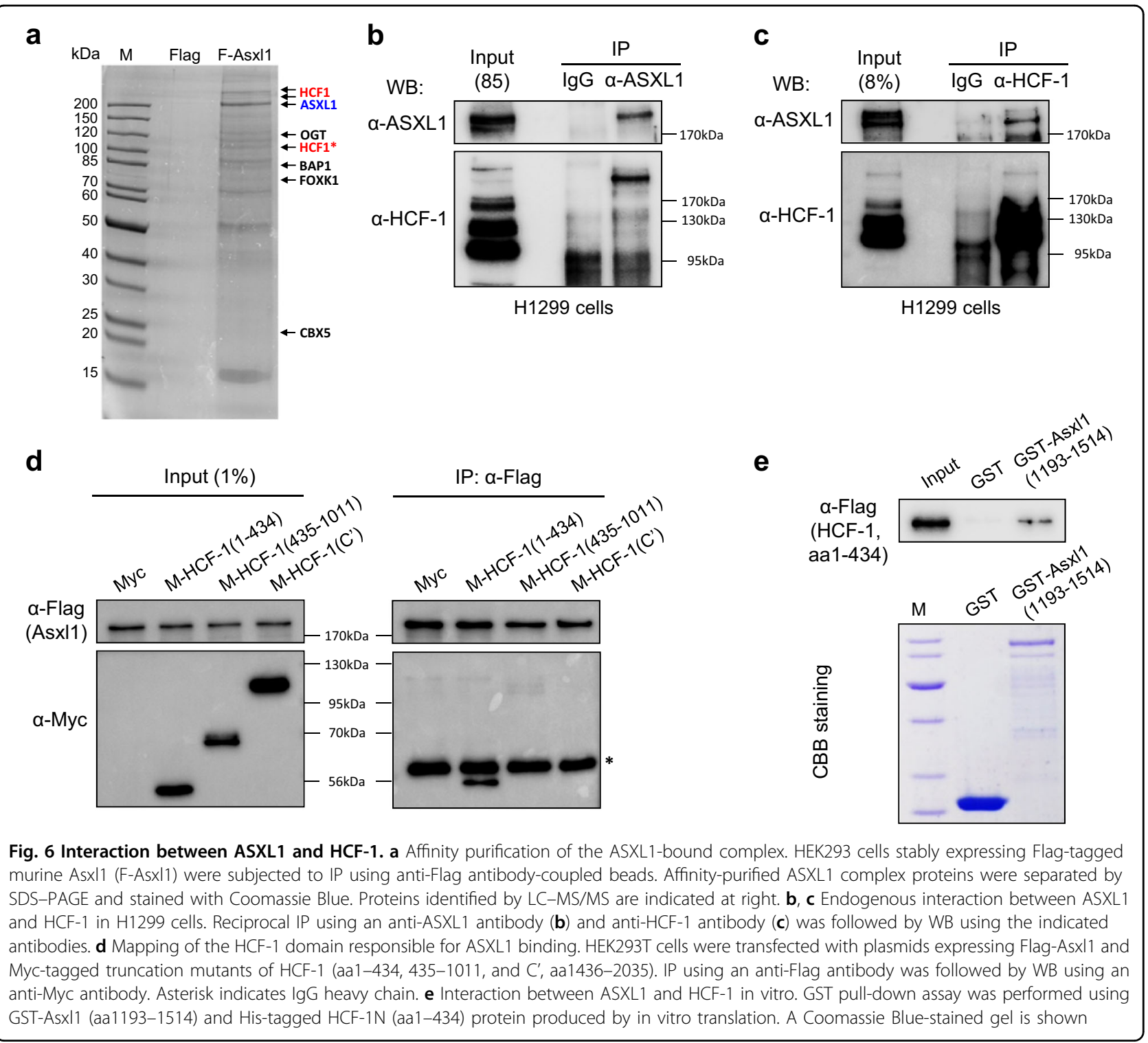

using an anti-Flag antibody was identified by liquid chromatography-tandem mass spectrometry analysis (Supplementary Table S4). Major binding partners, such as HCF-1, OGT, BAP1, FOXK1, and CBX5, were indicated in the Coomassie Blue-stained gel (Fig. 6a). Of these proteins, we focused on $\mathrm{HCF}-1$ as it binds to $\mathrm{E} 2 \mathrm{~F}^{32}$, and ASXL1 failed to bind to the E2F family (data not shown). Recently, HCF-1 was identified as an ASXL1-associated protein by affinity and size-exclusion chromatography ${ }^{23}$. However, it is unclear whether the interaction is direct and which portion is responsible for the interaction. The endogenous interaction between ASXL1 and HCF-1 in H1299 cells was demonstrated by two reciprocal co-immunoprecipitation (co-IP) assays followed by WB performed using antibodies specific for ASXL1 and HCF-1 (Fig. 6b, c). Additionally, the binding results were confirmed in E18.5 lung tissue and A549 cells, suggesting that this interaction is not cell-line specific (Supplementary Figures S6a, b). The functional domains of mAsxl1 and hHCF-1 are depicted in Supplementary Figures S6c, d. HCF-1 protein forms a heterodimer with HCF-1N and HCF-1C, cleaved products of the full-length precursor. To map the interaction region of HCF-1 with ASXL1, we generated three HCF-1 fragments with a Myc tag: amino acids (aa) 1-434, 435-1011, and 1436-2035. After transfection with Flag-Asxl1 into HEK293T cells, cell extracts were subjected to co-IP using an anti-Flag antibody. Subsequent WB using anti-Myc antibody indicated that the $\mathrm{N}$-terminal portion containing the Kelch domain of HCF-1 is responsible for its interaction with Asxl1 (Fig. 6d). Reciprocal mapping using GFP-tagged Asxl1 fragments and Flag-HCF-1 (aa1-434) showed that the 
C-terminal region (aa1193-1514), including the plant homeodomain (PHD) motif, is sufficient for the interaction with HCF-1 (Supplementary Figure S6e). Intriguingly, further co-IP using PHD-deleted Asxl1 indicated that no PHD motif is required for the interaction (data not shown). To determine whether the interaction is direct, a GST pull-down assay was performed using purified GST-fused Asxl1 (aa1193-1514) and in vitrotranslated HCF-1 (aa1-434) (Fig. 6e). These results suggest that ASXL1 directly interacts with HCF-1 through the $\mathrm{C}$-terminal region and the $\mathrm{N}$-terminal Kelch domain. Because HCF-1 functions as a coactivator of $\mathrm{E} 2 \mathrm{~F} 1^{32}$, we next evaluated whether HCF-1 is recruited to E2F1responsive promoters. For this purpose, we performed ChIP-qPCR using an anti-Flag antibody in HEK293T cells transiently transfected with Flag-tagged HCF-1N (aa1-434). As shown in Supplementary Figure S6f, HCF$1 \mathrm{~N}$ was detected on all E2F1-responsive sites of three E2F1 target genes. Further, we found that HCF-1N binds to the E2F1-binding locus of the Nmyc promoter in $\mathrm{H} 460$ and A549 cells (Supplementary Figure S6g). Overall, our data suggest that HCF-1, together with ASXL1, binds to E2F1-resposive promoters, including Nmyc, resulting in transcriptional repression.

\section{ASXL1 promotes the enrichment of $\mathrm{H} 3 \mathrm{~K} 27 \mathrm{me} 3$ at the E2F1-binding locus}

Given that ASXL1 is functionally associated with either EZH2, an H3K27 methyltransferase of the PRC2 com$\operatorname{plex}^{7,8}$, or with $\mathrm{HP} 1 \alpha$, a binder of methylated H3K 9 , for transcriptional silencing ${ }^{4}$, we addressed whether ASXL1, in cooperation with HCF-1, affects the enrichment of these epigenetic histone markers at the E2F1-binding locus of the Nmyc promoter. Upon overexpression of Asxl1 by adenoviral infection, H3K27me3 enrichment was enhanced at the E2F1-responsive sites of the Nmyc and CCNA2 promoters (Fig. 7a). In contrast, H3K4me3 enrichment was reduced on the promoters upon ASXL1 overexpression (Fig. 7b). These results were confirmed by ASXL1 depletion using adenoviral expression of
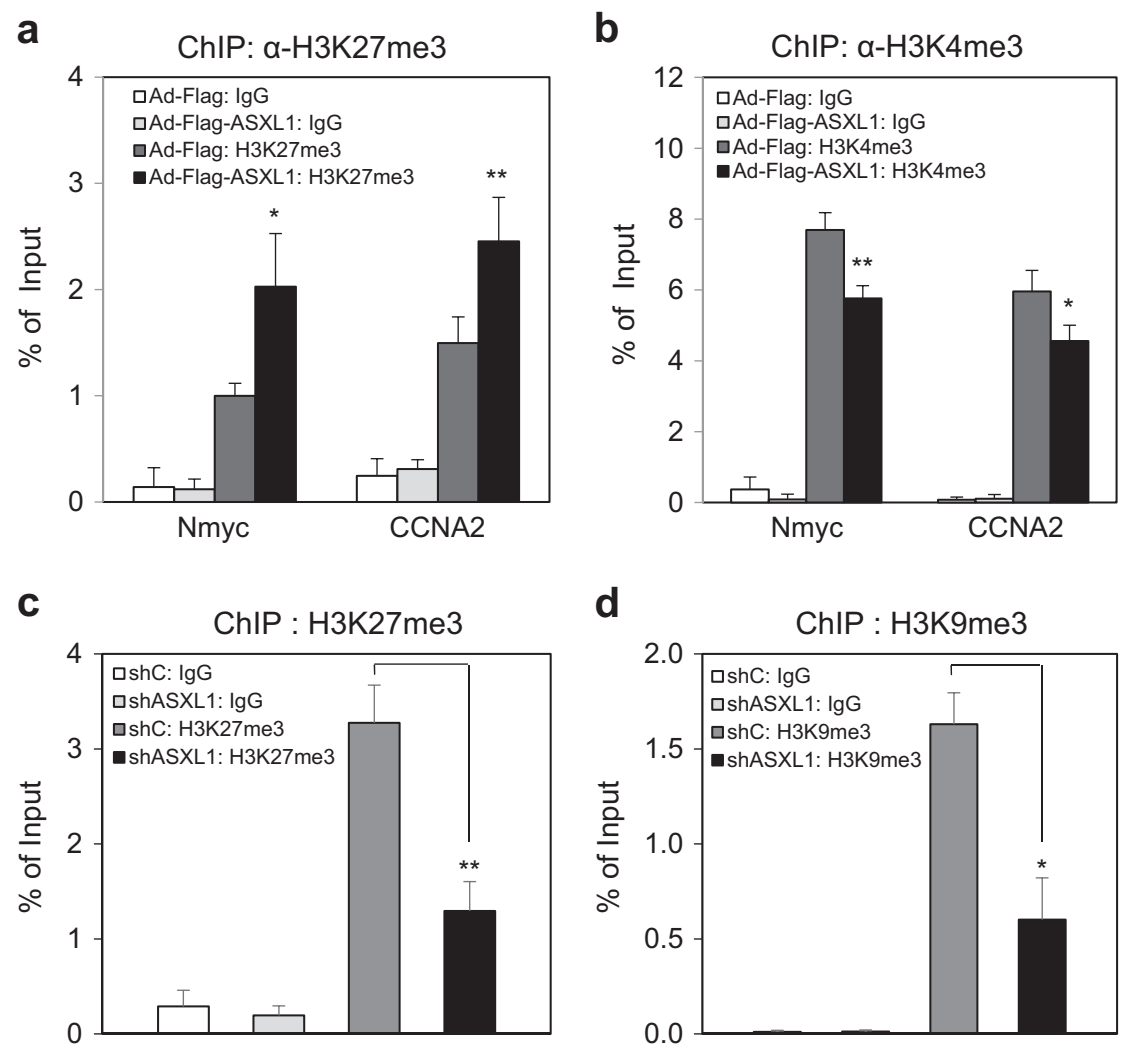

Fig. 7 Effect of ASXL1 on epigenetic histone modifications. Verification by ChIP-qPCR under ASXL1-overexpression and -knockdown conditions. IgG was used as a control for ChIP. qPCR was performed using site \#1 primer set as shown in Fig. 5. Data are means $\pm \operatorname{SD}\left(n=3,{ }^{*} P<0.05,{ }^{* *} P<0.01\right)$. $\mathbf{a}, \mathbf{b}$ Effect of Asxl1 overexpression on the enrichment of trimethylated histone H3 lysine 27 (H3K27me3) (a) and trimethylated H3 lysine 4 (H3K4me3) (b). A549 cells were infected with adenovirus-based Flag control or Flag-Asxl1 and subjected to ChIP-qPCR using an antibody against H3K27me3 or H3K4me3 and primer sets specific for the promoters of Nmyc and CCNA2. c, d Effect of ASXL1 depletion on the enrichment of H3K27me3 (c) and H3K9me3 (d). A549 cells were infected with adenovirus-based shLuciferase control (shC) or shASXL1 and subjected to ChIP-qPCR using an antibody against H3K27me3 or H3K9me3 and a primer set specific for the Nmyc promoter 
shASXL1. The level of $H 3 K 27 m e 3$ at the Nmyc promoter was significantly downregulated upon ASXL1 knockdown (Fig. 7c). Similarly, the promoter was less enriched in H3K9me3, another repressive histone marker (Fig. 7d), whereas $\mathrm{H} 3 \mathrm{~K} 9 \mathrm{ac}$, an active histone marker, was not affected (data not shown), thus leading to transcriptional derepression. Taken together, these data suggest that ASXL1 interacts with HCF-1 and suppresses the expression of Nmyc, an E2F1 target gene, by enriching H3K27me3 and H3K9me3 at the Nmyc promoter during normal fetal lung development.

\section{Discussion}

In this study, we demonstrated that murine Asxl1 is indispensable for neonatal survival and plays a role in pulmonary maturation and function. Histological analyses of lungs at different gestation stages showed that branching morphogenesis is normal in $A s x l 1^{-1-}$ embryos; however, abnormal saccular expansion was detected at the late gestation stage. Elevated proliferation of epithelial cells in Asxl1-null lungs appears to be associated with dysregulated expression of Ccsp (an airway epithelial marker) and Sox9 (a maker of distal epithelial progenitor cells). Subsequent analysis of Type I and II alveolar pneumocytes revealed that Asxl1 loss increases proliferation of immature cells but decreases differentiation of these cells for maturation (Fig. 8), which is partly consistent with previous report ${ }^{22}$. The reduced surfactant production and excessive glycogen content associated with defective phospholipid synthesis caused by the absence of Asxl1 in Type II cells leads to respiratory failure and poor neonatal mortality ${ }^{27}$. Various factors, such as coactivator-associated arginine methyltransferase 1 (CARM1), Foxa2, Foxm1, and Mst1/2, are required for proper alveolar formation during fetal lung development $^{33-36}$. Disruption of these genes leads to fetal lethality due to a RDS-like phenotype ${ }^{37}$, which includes lung collapse. As this phenotype is similar to that of the lungs of As $x l 1^{-1-}$ mice, our $A s x l 1^{-1-}$ mice may represent a new model for the RDS-like phenotype. Next, lung epithelialspecific Asxl1 conditional KO mice need to be generated by crossing with $N k x 2.1$-Cre or Shh-Cre mice. Additionally, analysis of ASXL1 mutations in human RDS patients by exome sequencing is warranted.

A role for ASXL1 as a tumor suppressor was suggested by the altered gene expression by Asxl1 deletion and the upregulation of genes involved in cell-cycle progression (e.g., E2F1 target genes such as Nmyc). The genes upregulated by Asxl1 KO are associated with poor survival (Supplementary Figure S7a), and the genes downregulated by Asxl1 $\mathrm{KO}$ are associated with good survival in lung cancer patients (Supplementary Figure S7b). Survival analysis using two lung cancer databases revealed better survival when ASXL1 expression was higher (Supplementary Figure S7c). Lung cancer patients with high levels of ASXL1 expression and lower levels of NMYC

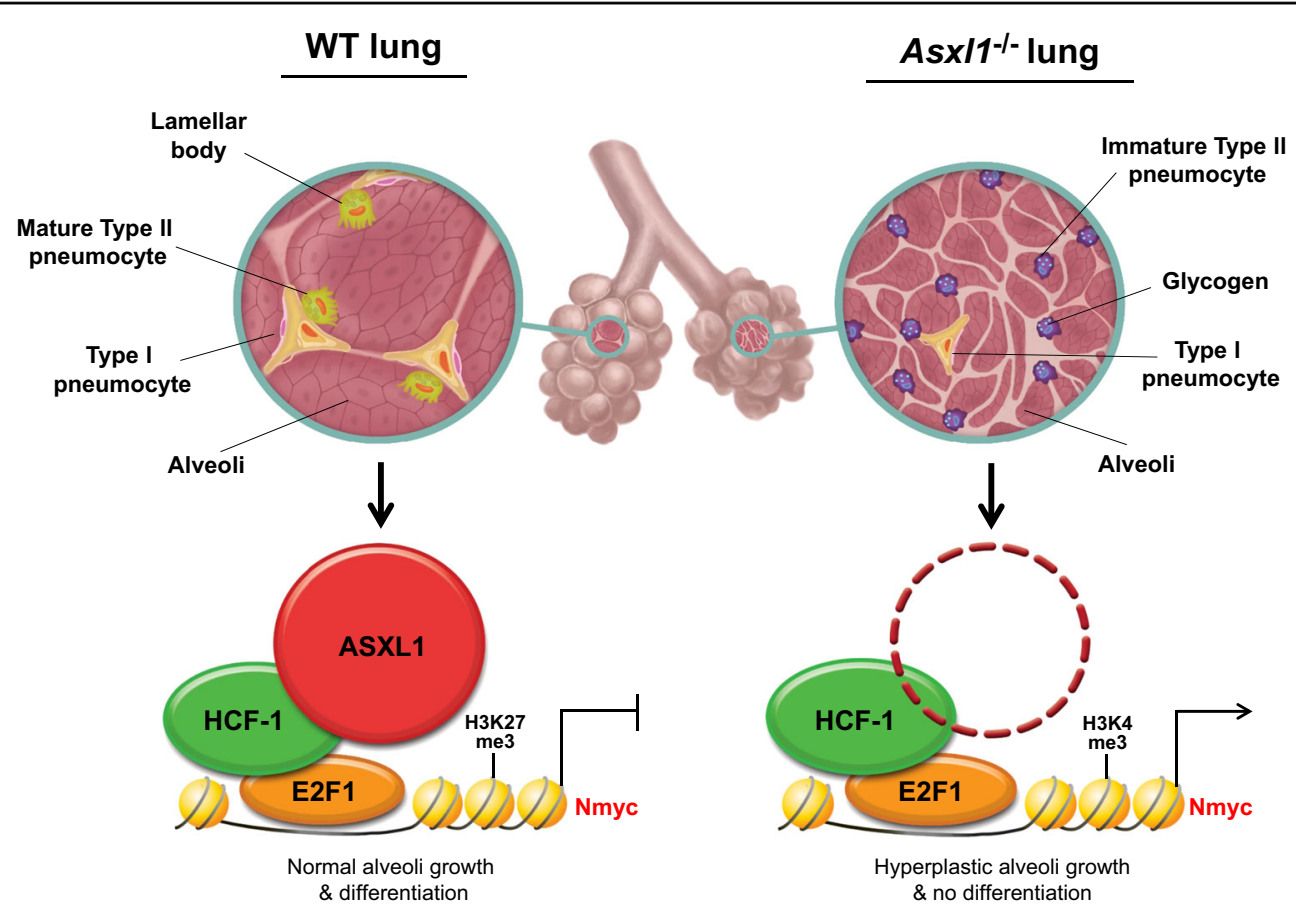

Fig. 8 Hypothetical model for the epigenetic role of Asxl1 in regulating Nmyc expression during mouse fetal lung development. Details are provided in the Discussion section 
expression showed better survival (Supplementary Figure S7d), suggesting a close link between ASXI1 and NMYC. In addition to their role in progenitor cell proliferation during fetal lung development ${ }^{30}$, NMYC and its transcriptional regulator E2F1 are amplified and upregulated, respectively, in small-cell lung cancer ${ }^{38,39}$. According to our hypothesis, Asxl1 deletion may promote progenitor cell proliferation and repress their differentiation for alveolar maturation by increasing Nmyc expression, leading to hyperproliferation of premature alveolar epithelial cells, which initiates lung tumorigenesis. To test this hypothesis, lung-specific conditional deletion of Asxl1 will be required to avoid the postnatal lethality caused by constitutive knockout. In contrast to the antiproliferative role of Asxl1 in lung development, our previous study indicated that Asxl1 interacts with Akt and Ezh2 in mouse embryonic fibroblasts to maintain cell cycle progression, and Asxl1 disruption leads to cellular senescence ${ }^{8}$. Therefore, we propose that ASXL1 regulates cell proliferation positively or negatively depending on the input signals during development.

A microarray analysis and subsequent validation by RTqPCR indicated that Asxl1 represses the expression of E2F1-responsive genes, including Nmyc. To investigate the mechanism underlying this repression, we determined whether ASXL1 directly interacts with the E2F family; no interaction was detected (data not shown). BAP1 binds to E2F1-responsive gene promoters through HCF-1 and upregulates their expression, and BAP1 KO inhibits the growth of uveal melanoma cells ${ }^{40,41}$. However, no direct evidence of ternary complex formation by E2F1, HCF-1, and BAP1 was provided. Likewise, we identified HCF-1 as an ASXL1-binding protein and mapped the interaction sites between the C-terminal region of ASXL1 and the Nterminal Kelch domain of HCF-1 (Fig. 8). In vitro GST pull-down assays showed that the interaction is direct, not via BAP1, and this was confirmed by co-IP using a BAP1binding-defective ASXL1 mutant (data not shown). As a transcriptional coregulator, HCF-1 associates with E2F1 and localizes to E2F1-responsive promoters to activate E2F1 by recruiting the histone H3K4 methyltransferase, mixed-lineage leukemia (MLL) ${ }^{32}$. Like other E2F1responsive genes, E2F1 binds to the proximal Nmyc promoter and modulates its expression ${ }^{29}$. The role of murine Nmyc at the molecular level in fetal lung development has been mapped using mouse models and genome-wide approaches $^{30,31}$. Thus, our studies focused on the epigenetic roles of ASXL1 and HCF-1 in the repression of E2F1 target genes, including Nmyc (Fig. 8). ASXL1 increased the level of the repressive histones H3K27me3 and H3K9me3 at the E2F1-responsive site. In contrast, the level of active H3K4me3 was reduced, implying that ASXL1 interacted with HCF-1 and may have interfered with its activation of E2F1 through the H3K4 methyltransferase MLL. Questions about whether ASXL1 affects the interaction between HCF-1 and MLL remain to be answered. Additionally, it will be of interest to address whether BAP1 plays a role in Nmyc repression by forming a complex with ASXL1 and HCF-1, and by deubiquitinating histone $\mathrm{H} 2 \mathrm{AK} 119$ which is monoubiquitinated by PRC1. Our findings provide considerable insight into the tumor-suppressive role of ASXL1 in lung cancer and will facilitate the development of novel diagnosis and treatment strategies of pulmonary disorders including respiratory distress syndrome.

\section{Materials and methods \\ Mouse strains and breeding}

Asxl1-deficient mice (Asxl1 < tm1a(EUCOMM)Wtsi>, MGI ID:2684063, EMMA ID:03996) have been described previously $^{21}$. All animal experiments were performed according to the regulations of the Korean Council on Animal Care, and all protocols were reviewed by the Sejong Animal Care Committee.

\section{Cell lines and cell culture}

The HEK293T, A549, and H460 cell lines were obtained from the American Type Culture Collection (ATCC, Manassas, VA, USA). HEK293T cells were cultured in Dulbecco's modified Eagle's medium (DMEM) supplemented with $10 \%$ fetal bovine serum (FBS) (GenDEPOT) and an antibiotic-antimycotic (Gibco) in a $5 \% \mathrm{CO}_{2}$ atmosphere at $37^{\circ} \mathrm{C}$. A549 and $\mathrm{H} 460$ cells were cultured in the RPMI-1640 medium supplemented with 10\% FBS and an antibiotic-antimycotic in a $5 \% \mathrm{CO}_{2}$ atmosphere at $37^{\circ} \mathrm{C}$.

\section{Plasmids and cloning}

All cDNAs were constructed according to standard methods and verified by sequencing. Flag-tagged $(2 \times)$ mAsxl1 and Myc-tagged hHCF-1 deletion genes were ligated into the pcDNA3 vector (Invitrogen). GFP-tagged deletion constructs were ligated into pEGFP-C3 (BD Biosciences) ${ }^{21}$. For GST-fused proteins, pGEX4T-1 (GE Healthcare) was used.

\section{Immunohistochemistry}

For immunohistochemistry, mouse embryonic lung tissues were collected in ice-cold $1 \times$ PBS and fixed in $4 \%$ paraformaldehyde overnight at $4{ }^{\circ} \mathrm{C}$. Fixed lungs were washed with $1 \times$ PBS, gradually postfixed in 15 and $30 \%$ sucrose at $4{ }^{\circ} \mathrm{C}$ to impregnate fully, and embedded in Tissue-Tek OCT compound (Sakura Finetek, Japan). Histological analysis was performed on $6 \mu \mathrm{m}$ sections. Hematoxylin and eosin (H\&E), PAS, and SBB staining were performed according to standard protocols ${ }^{42,43}$. Sections were processed for antigen retrieval in $0.01 \mathrm{M}$ citric acid solution ( $\mathrm{pH}$ 6.0) for $15 \mathrm{~min}$. After blocking 
with blocking solution (5\% horse serum, 3\% bovine serum albumin, $0.1 \%$ Triton X-100 in $1 \times \mathrm{PBS}$ ) for $1 \mathrm{~h}$, the slides were incubated with primary antibodies in blocking solution overnight at $4{ }^{\circ} \mathrm{C}$. The primary antibodies used were Syrian hamster anti-pdpn (1:100; Santa Cruz, sc53533), rabbit anti-proSP-C (1:200; Chemicon, 50-173573), goat anti-CC10 (1:200; Santa Cruz, sc-9772), mouse anti- $\alpha$ SMA (1:300; Santa Cruz, sc-53142), mouse antiPCNA (1:200; Santa Cruz, sc-56), and rabbit anti-SP-B (1:200; Millipore, AB3430). The slides were washed with $1 \times$ PBS and incubated with Alexa 488 goat anti-rabbit IgG at a 1:200 dilution, Alexa 568 goat anti-mouse IgG at a 1:200 dilution, Alexa 568 donkey anti-goat IgG at a 1:200 dilution, and Alexa 568 goat anti-hamster IgG at a 1:200 dilution (Invitrogen). The cover glasses were mounted using Vectashield Mounting Medium (Vector, H-1000).

\section{Radio and RNAscope in situ hybridization}

Radio in situ hybridization (ISH) was performed as described previously ${ }^{44}$. Hybridization probes were generated from the subcloned pcDNA3.1 plasmids using the following primers specific for murine Asxl1: forward, XhoI site-ggg CTC GAG TAC AGA GTC TCA GAG CCG AC; reverse, BamHI site-ggg GGA TCC GTT GCT GGA AGT GTA GT. The primers used for the murine Nmyc probe were: forward, XhoI site-ggg CTC GAG CCT GGG TGG CCT CAC TCC TA; reverse, BamHI site-ggg GGA TCC CGC TCA AGG TAT CCT CTC CGG. For RNAscope ISH, E18.5 mice were euthanized, and their lungs frozen immediately. RNAscope is commercially available from advanced cell diagnostics (ACD). Hybridization was performed according to the RNAscope ${ }^{\circledR} 2.0$ HD Detection kit (Brown) User Manual (ACD, \#320497). In brief, lungs were embedded in optimum cutting temperature compound, and sections were thaw-mounted onto Superfrost Plus Microscope Slides (Fisher Scientific). The murine Asxl1 (Cat No. 421961-C2) mRNA target probe was manufactured by ACD. The sections were fixed in $4 \%$ paraformaldehyde for $10 \mathrm{~min}$; dehydrated in 50, 75, 95, and $100 \% \mathrm{EtOH}$ for $5 \mathrm{~min}$; and air-dried. Tissue was treated with pretreatment solution for $10 \mathrm{~min}$ at room temperature (RT). For RNA detection, tissue was incubated with the Asxl1 probe for $2 \mathrm{~h}$ at $40^{\circ} \mathrm{C}$, and the amplifier solutions were added. Each amplifier was removed by washing using washing buffer $(1 \times)$ for $2 \mathrm{~min}$ at RT. The signals were developed with DAB with hematoxylin counterstaining. The slides were washed, mounted, and viewed under a Leica fluorescence microscope (DFC420 C).

\section{RNA isolation, reverse transcription, and RTqPCR}

RNA isolation and qPCR were carried out as reported previously ${ }^{21}$ using the primers listed in Supplementary Table S5. All expression levels were normalized to that of
GAPDH, and results were plotted as relative to WT lungs or empty controls.

\section{Microarray analysis}

Total RNA $(10 \mu \mathrm{g})$ isolated from E18.5 $\mathrm{Asxl1} \mathrm{1}^{-1-}$ and WT lungs was subjected to analysis with the Agilent Bioanalyzer System (Mouse GE 4× 44 K v2 Microarray: Agilent, Santa Clara, CA, USA) according to the manufacturer's instructions. Asxl1 $1^{-1-}$ signal intensities were normalized to those of the WT. Results were filtered, and the cut off was set at a twofold difference. Genes exhibiting significant differences in expression levels were classified into Gene Ontology (GO) based functional categories (http://www.geneontology.org) using the PANTHER (http://www.pantherdb.org), KEGG (http://www.genome.jp/kegg), DAVID (http://david.abcc. ncifcrf.gov), and Medline (http://www.ncbi.nlm.nig.gov) databases.

\section{Western blotting and co-immunoprecipitation}

WB and co-IP were carried out as reported previously ${ }^{21}$. Briefly, lungs were homogenized in lysis buffer $(50 \mathrm{mM}$ Tris-Cl, pH 7.5, $150 \mathrm{mM} \mathrm{NaCl}, 0.5 \%$ Nonidet P-40, $5 \mathrm{mM}$ EDTA, $1 \mathrm{mM}$ PMSF) supplemented with protease inhibitors (Roche). Generally, 40-60 $\mu \mathrm{g}$ protein-containing supernatants were separated by electrophoresis in $10 \%$ sodium dodecyl sulfate polyacrylamide gels, transferred to nitrocellulose membranes, and incubated with the indicated primary antibodies: rabbit anti-ASXL1 (GeneTex Inc., GTX127284), anti-HCF-1 (Bethyl Laboratories, A301-399A), mouse anti-Flag (MBL International, FLA-1), mouse anti-Myc (MBL International, My3), mouse anti-GFP (Santa Cruz, sc-9996), and mouse anti- $\beta$ actin (Santa Cruz, sc-47778). The blots were incubated with peroxidase-conjugated secondary antibodies (goat anti-rabbit IgG, goat anti-mouse IgG, and goat anti-Syrian hamster IgG, as appropriate; Santa Cruz). The protein bands were detected using ECL reagent (Intron) followed by exposure to X-ray film (Agfa) or detection in a Vilber Fusion Solo 2 Chemiluminescence System (Fisher Biotec). For IP, we used a rabbit anti-ASXL1 polyclonal antibody raised against aa 233-247 of mouse Asxl1 ${ }^{3}$.

\section{Construction of the Nmyc promoter-luciferase reporter}

The Nmyc promoter $(-3.2 \mathrm{~kb}$ in length) generated by mouse tail gDNA was subcloned upstream of a luciferase reporter gene into the pGL2-basic vector (Promega). The 5'-deletion and E2F1-binding site mutants in the Nmyc promoter were produced by PCR. Primers were designed with Nhel and $\mathrm{BamHl}$ sites at the ends of the products (see Supplementary Table S5). All plasmids were verified by restriction enzyme digestion and DNA sequencing. Luciferase activity was measured as described previously ${ }^{3}$. 


\section{Recombinant adenovirus}

For overexpression in mammalian cells, Asxl1 adenovirus was generated using the pAdEasy system ${ }^{45}$. Flagtagged mAsxl1 was subcloned into the pAdTrackCMV plasmid and recombined with the pAdEasy adenovirus backbone plasmid in E. coli BJ5183. QBI-293A cells were transfected with recombinant plasmid, and the virus was amplified. The virus titer was measured using an Adeno- $\mathrm{X}$ Rapid Titer Kit (Clontech). For KO in mammalian cells, recombinant adenovirus-expressing shRNA was prepared. The duplex DNA effective for both human and mouse ASXL1 (Supplementary Table S5) was digested with Notl and subcloned into the digested PBS/U6 vector (Addgene). The U6 promoter-driven shASXL1 was excised and ligated into the pAdtrack vector (Addgene). The pAdTrack-U6 shASXL1 obtained was recombined with pAdEasy-1 by transformation in E. coli BJ5183. Recombinant adenovirus was produced by transfecting the recombinant plasmid into QBI-293A cells. Infection and $\mathrm{KO}$ efficiency were monitored by GFP fluorescence and RT-qPCR, respectively. To evaluate the optimal multiplicity of infection (MOI) for maximal infection and transgene expression, $60-\mathrm{mm}$ dishes containing $2 \times 10^{5}$ A549 cells were infected with adenovirus at MOIs of 50, 100 , and 200 for $24 \mathrm{~h}$. Adenoviral infection efficiency was assessed based on GFP expression. Asxl1 expression in A549 cells was evaluated by RT-PCR and WB.

\section{Glutathione S-transferase pull-down assay}

Glutathione S-transferase (GST) pull-down assay was performed as described previously ${ }^{3}$. GST-fused Asxl1 (aa1193-1514) protein was expressed in E. coli and purified using glutathione-Sepharose beads (GE Healthcare). Flag-HCF-1 (aa1-434) protein was translated in vitro using a TNT $^{\circ}$ rabbit reticulocyte system (Promega). Briefly, $2 \mu \mathrm{g}$ of GST-Asxl1 (or GST) was incubated with $10 \mu \mathrm{L}$ of Flag-HCF-1 protein. Bound protein was visualized by WB using an anti-Flag M2 monoclonal antibody (Sigma, F-3165).

\section{Chromatin immunoprecipitation}

ChIP assay was performed as described previously with some modifications ${ }^{46}$. A549 and $\mathrm{H} 460$ cells were transfected with the Flag-E2F1 expression plasmid. Crosslinked sheared chromatin complexes were recovered by IP using the antibodies indicated, and the cross-linking was reversed. The released DNA pellets were amplified by quantitative PCR using a primer pair that encompassed the E2F1-binding sites in the Nmyc, CDC6, CCNA2, and $C D C 25 A$ promoter regions (Supplementary Table S5). Antibodies used for histone modifications are mouse antiH3K4me3 (Millipore, 07-473), mouse anti-H3K9me3 (Millipore, 07-442), mouse anti-H3K27me3 (Abcam, ab6002; Millipore, 07-449).

\section{TUNEL assay}

In situ cell death was evaluated using a TUNEL assay kit (Roche). Lungs were fixed, permeabilized, and incubated in TUNEL reagent micro-drops comprising $10 \%$ of the enzymatic solution (deoxynucleotidyl terminal transferase) and $90 \%$ of the marking solution (2'-deoxyuridine $5^{\prime}$-triphosphate-DUTP + fluorescein isothiocyanate-conjugated-FITC) for $1 \mathrm{~h}$ in a humid chamber at $37^{\circ} \mathrm{C}$ in the dark. The positive control was a sample treated with 1 $\mathrm{IU} / \mu \mathrm{L}$ DNase (Promega); the negative control was incubated in micro-drops containing only marking solution. After washing, samples were stained with Hoechst 33342 (Sigma), and the cover glasses were mounted with Vectashield Mounting Medium (Vector, H-1000).

\section{Purification of ASXL1-associated protein complex}

HEK293 cells stably expressing Flag-tagged Asxl1 or Flag vector were grown in $20 \times 150 \mathrm{~cm}$ dishes. Affinity purification of the ASXL1 protein complex was carried out as reported previously ${ }^{44}$.

\section{Statistical analysis}

Data are means \pm standard deviation of at least three independent experiments. Comparisons between multiple groups were performed by paired Student's $t$ tests. $P$ values $<0.05\left(^{(")}\right), 0.01\left(^{* * *}\right)$, or $0.001\left(^{(* * * * *}\right)$ were considered to indicate statistical significance.

\section{Acknowledgments \\ We thank Center for NeuroGenomics in Korea University for technical support for In Situ Hybridization and In-Hee Yang for assisting graphic summary. This study was supported in part by a grant from the Korea Food Research Institute funded by the Ministry of Science, ICT \& Future Planning (E0150301-04) and a grant of the Bio \& Medical Technology Development Program of the NRF funded by the Korean government, MSIT (2018M3A9H1023139). \\ Author details \\ ${ }^{1}$ Department of Integrative Bioscience and Biotechnology, Sejong University, Seoul 05006, Korea. ${ }^{2}$ Severance Biomedical Science Institute, Yonsei University College of Medicine, Seoul 06273, Korea. ${ }^{3}$ Department of Anatomy, Korea University College of Medicine, Seoul 02841, Korea. ${ }^{4}$ School of Biological Science, College of Natural Sciences, Seoul National University, Seoul 08826, Korea. ${ }^{5}$ Korea Food Research Institute, Jeonju, Jeonbuk 55365, Korea. ${ }^{6}$ Department of Molecular Biology, Dankook University, Chungnam 31116 , Korea}

\section{Authors' contributions}

S.M. and S.J.U. designed the studies. S.M., N.K., J.Y.K. and Y.K.L. conducted the experiments. U.H.P., H.Y. and E.J.K. analyzed and interpreted microarray data. A.R.K. and S.J.A. maintained Asx/1-null mice and prepared recombinant adenovirus. S.K.I., J.H.K., J.T.H. and W.S. provided technical assistance for immunocytochemistry and in situ hybridization. S.M. and S.J.U. analyzed the data and wrote the manuscript.

Conflict of interest

The authors declare that they have no conflict of interest.

\section{Publisher's note}

Springer Nature remains neutral with regard to jurisdictional claims in published maps and institutional affiliations. 
Supplementary Information accompanies this paper at (https://doi.org/ 10.1038/s41419-018-1171-z).

Received: 13 July 2018 Revised: 4 October 2018 Accepted: 19 October 2018 Published online: 02 November 2018

\section{References}

1. Milne, T. A., Sinclair, D. A. \& Brock, H. W. The additional sex combs gene of Drosophila is required for activation and repression of homeotic loci, and interacts specifically with Polycomb and super sex combs. Mol. Gen. Genet. 261, 753-761 (1999)

2. Katoh, M. Functional and cancer genomics of ASXL family members. Br. J. Cancer 109, 299-306 (2013).

3. Cho, Y. S., Kim, E. J., Park, U. H., Sin, H. S. \& Um, S. J. Additional sex comb-like1 (ASXL1), in cooperation with SRC-1, acts as a ligand-dependent coactivator for retinoic acid receptor. J. Biol. Chem. 281, 17588-17598 (2006).

4. Park, U. H., Yoon, S. K., Park, T., Kim, E. J. \& Um, S. J. Additional sex comb-like (ASXL) proteins 1 and 2 play opposite roles in adipogenesis via reciprocal regulation of peroxisome proliferator-activated receptor \{gamma\}. J. Biol. Chem. 286, 1354-1363 (2011).

5. Scheuermann, J. C. et al. Histone H2A deubiquitinase activity of the polycomb repressive complex PR-DUB. Nature 465, 243-247 (2010).

6. Sahtoe, D. D., van Dijk, W. J., Ekkebus, R., Ovaa, H. \& Sixma, T. K. BAP1/ASXL1 recruitment and activation for $\mathrm{H} 2 \mathrm{~A}$ deubiquitination. Nat. Commun. 7, 10292 (2016).

7. Abdel-Wahab, $\mathrm{O}$. et al. ASXL1 mutations promote myeloid transformation through loss of PRC2-mediated gene repression. Cancer Cell. 22, 180-193 (2012).

8. Youn, H. S. et al. Asxl1 deficiency in embryonic fibroblasts leads to cellular senescence via impairment of the AKT-E2F pathway and Ezh2 inactivation Sci. Rep. 7, 5198 (2017)

9. Gelsi-Boyer, V. et al. Mutations in ASXL1 are associated with poor prognosis across the spectrum of malignant myeloid diseases. J. Hematol. Oncol. 5, 12 (2012).

10. Micol, J. B. \& Abdel-Wahab, O. The role of additional sex combs-like proteins in cancer. Cold Spring Harb. Perspect. Med. 6, a026526 (2016).

11. Oak, J. S. \& Ohgami, R. S. Focusing on frequent ASXL1 mutations in myeloid neoplasms, and considering rarer ASXL2 and ASXL3 mutations. Curr. Med. Res. Opin. 33, 781-782 (2017)

12. Abdel-Wahab, O. et al. Deletion of Asxl1 results in myelodysplasia and severe developmental defects in vivo. J. Exp. Med. 210, 2641-2659 (2013).

13. Inoue, D. et al. Myelodysplastic syndromes are induced by histone methylation-altering ASXL1 mutations. J. Clin. Invest. 123, 4627-4640 (2013).

14. Wang, J. et al. Loss of Asxl1 leads to myelodysplastic syndrome-like disease in mice. Blood 123, 541-553 (2014)

15. Yang, $H$. et al. Gain of function of ASXL1 truncating protein in the pathogenesis of myeloid malignancies. Blood 131, 328-341 (2018).

16. Dey, A. et al. Loss of the tumor suppressor BAP1 causes myeloid transformation. Science 337, 1541-1546 (2012).

17. LaFave, L. M. et al. Loss of BAP1 function leads to EZH2-dependent transformation. Nat. Med. 21, 1344-1349 (2015)

18. Hoischen, A. et al. 2011. De novo nonsense mutations in ASXL1 cause Bohring-Opitz syndrome. Nat. Genet. 43, 729-731 (2011).

19. Dangiolo, S. B., Wilson, A., Jobanputra, V. \& Anyane-Yeboa, K. Bohring-Optiz syndrome (BOS) with a new ASXL1 pathogenic variant: review of the most prevalent molecular and phenotypic features of the syndrome. Am. J. Med. Genet. A 167A, 3161-3166 (2015)

20. Fisher, C. L. et al. Additional sex combs-like 1 belongs to the enhancer of trithorax and polycomb group and genetically interacts with Cbx2 in mice. Dev. Biol. 337, 9-15 (2010).

21. Moon, S., Um, S. J. \& Kim, E. J. Role of Asxl1 in kidney podocyte development via its interaction with Wtip. Biochem. Biophys. Res. Commun. 466, 560-566 (2015).
22. McGinley, A. L., Li, Y., Deliu, Z. \& Wang, Q. T. Additional sex combs-like family genes are required for normal cardiovascular development. Genesis $\mathbf{5 2}$ 671-686 (2014)

23. Wu, X et al. Tumor suppressor ASXL1 is essential for the activation of INK4B expression in response to oncogene activity and anti-proliferative signals. Cell Res. 25, 1205-1218 (2015)

24. Berger, J. \& Bhandari, V. Animal models of bronchopulmonary dysplasia. The term mouse models. J. Physiol. Lung Cell Mol. Physiol. 307, L936-L947 (2014).

25. Turgeon, B. \& Meloche, S. Interpreting neonatal lethal phenotypes in mouse mutants: insights into gene function and human diseases. Physiol. Rev. 89 $1-26$ (2009)

26. Warburton, D. et al. The molecular basis of lung morphogenesis. Mech. Dev 92, 55-81 (2000).

27. Rockich, B. E. et al. Sox9 plays multiple roles in the lung epithelium during branching morphogenesis. Proc. Natl. Acad. Sci. USA 110, E4456-E4464 (2013).

28. Whitsett, J. A. \& Weaver, T. E. Hydrophobic surfactant proteins in lung function and disease. N. Engl. J. Med. 347, 2141-2148 (2002).

29. Strieder, V. \& Lutz, W. E2F proteins regulate MYCN expression in neuroblastomas. J. Biol. Chem. 278, 2983-2989 (2003)

30. Okubo, T., Knoepfler, P. S., Eisenman, R. N. \& Hogan, B. L. Nmyc plays an essential role during lung development as a dosage sensitive regulator of progenitor cell proliferation and differentiation. Development 132, 1363-1374 (2005).

31. Cox, B. et al. Integrated proteomic and transcriptomic profiling of mouse lung development and Nmyc target genes. Mol. Syst. Biol. 3, 109 (2007).

32. Tyagi, S., Chabes, A. L., Wysocka, J. \& Herr, W. E2F Activation of S phase promoters via association with HCF-1 and the MLL family of histone H3K4 methyltransferases. Mol. Cell 27, 107-119 (2007)

33. Wan, H. et al. Foxa2 is required for transition to air breathing at birth. Proc. Nat Acad. Sci. USA 101, 14449-14454 (2004).

34. Kalin, T. V. et al. Forkhead Box $\mathrm{m} 1$ transcription factor is required for perinatal lung function. Proc. Natl Acad. Sci. USA 105, 19330-19335 (2008).

35. O'Brien, K. B et al. CARM1 is required for proper control of proliferation and differentiation of pulmonary epithelial cells. Development 137, 2147-2156 (2010).

36. Chung, C. et al. Hippo-Foxa2 signaling pathway plays a role in peripheral lung maturation and surfactant homeostasis. Proc. Natl Acad. Sci. USA 110 7732-7737 (2013)

37. Whitsett, J. A., Wert, S. E. \& Weaver, T. E. Alveolar surfactant homeostasis and the pathogenesis of pulmonary disease. Annu. Rev. Med. 61, 105-119 (2010).

38. Eymin, B., Gazzeri, S., Brambilla, C. \& Brambilla, E. Distinct pattern of E2F1 expression in human lung tumours: E2F1 is upregulated in small cell lung carcinoma. Oncogene 14, 1678-1687 (2001).

39. Blanco, R. et al. A gene-alteration profile of human lung cancer cell lines. Hum Mutat. 30, 1199-1206 (2009).

40. Eletr, Z. M. \& Wilkinson, K. D. An emerging model for BAP1's role in regulating cell cycle progression. Cell Biochem. Biophys. 60, 3-11 (2011).

41. Pan, $\mathrm{H}$. et al. BAP1 regulates cell cycle progression through $\mathrm{E} 2 \mathrm{~F} 1$ target genes and mediates transcriptional silencing via $\mathrm{H} 2 \mathrm{~A}$ monoubiquitination in uveal melanoma cells. Int. J. Biochem. Cell. Biol. 60, 176-184 (2015).

42. Xu, B. et al. Smad1 and its target gene Wif1 coordinate BMP and Wnt signaling activities to regulate fetal lung development. Development 138,925-935 (2011).

43. Yamazaki, D. et al. Essential role of the TRIC-B channel in $\mathrm{Ca}+$ handling of alveolar epithelial cells and in perinatal lung maturation. Development $\mathbf{1 3 6}$ 2355-2361 (2009).

44. Choe, J. et al. Effect of thymosin beta15 on the branching of developing neurons. Biochem. Biophys. Res. Commun. 331, $43-49$ (2005).

45. He, T. C. et al. A simplified system for generating recombinant adenoviruses. Proc. Natl Acad. Sci. USA 95, 2509-2514 (1998).

46. Park, U. H. et al. ASXL2 promotes proliferation of breast cancer cells by linking ERa to histone methylation. Oncogene 28, 3742-3752 (2016). 